\title{
TRANSFORMATIONS ÉNERGÉTIQUES SOUS CONTRAINTE ÉCOLOGIQUE FORTE
}

\author{
Alain Nadaï, Grégoire Wallenborn
}

Association Multitudes | « Multitudes »

2019/4 n $77 \mid$ pages 43 à 53

ISSN 0292-0107

Article disponible en ligne à l'adresse :

https://www.cairn.info/revue-multitudes-2019-4-page-43.htm

Distribution électronique Cairn.info pour Association Multitudes.

(C) Association Multitudes. Tous droits réservés pour tous pays.

La reproduction ou représentation de cet article, notamment par photocopie, n'est autorisée que dans les limites des conditions générales d'utilisation du site ou, le cas échéant, des conditions générales de la licence souscrite par votre établissement. Toute autre reproduction ou représentation, en tout ou partie, sous quelque forme et de quelque manière que ce soit, est interdite sauf accord préalable et écrit de l'éditeur, en dehors des cas prévus par la législation en vigueur en France. Il est précisé que son stockage dans une base de données est également interdit. 

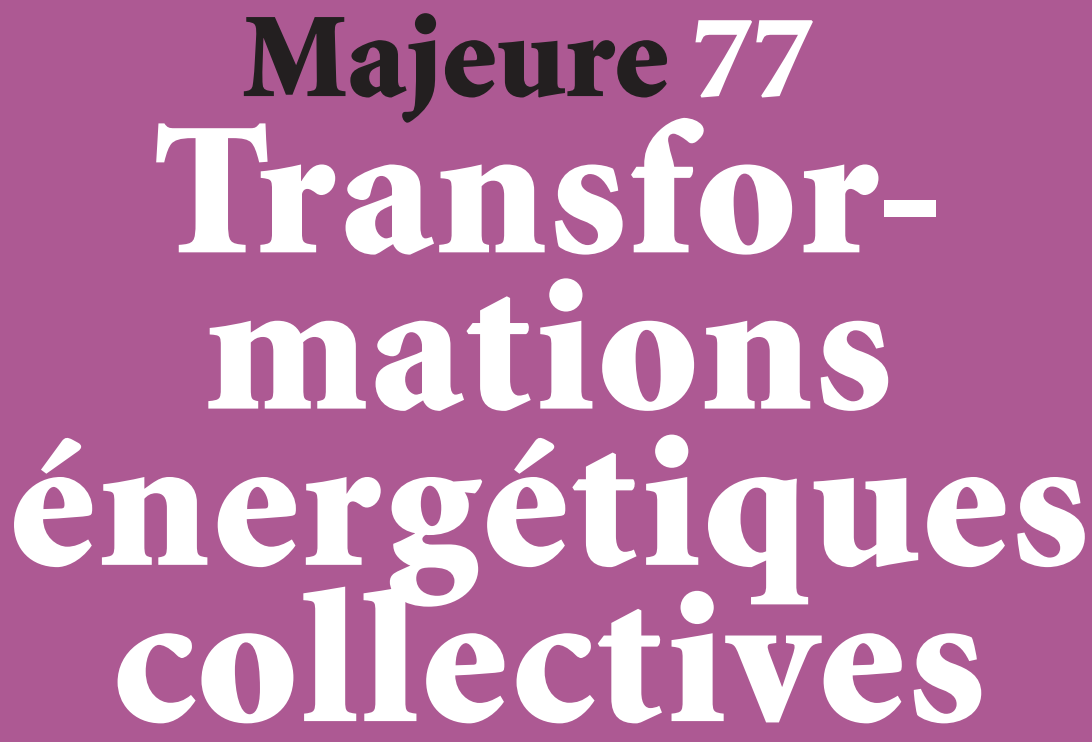

L'urgente nécessité de contenir les effets du changement climatique appelle des transformations énergétiques radicales. À partir de quelques expériences en cours, en France (fermes solaires en Rhône-Alpes) et ailleurs (éoliennes en Frise du Nord, gaz naturel à Istanbul, solaire photovoltaïque au Cap), cette majeure s'interroge sur ce qui favorise ces transformations, ce qu'elles requièrent et quels sont leurs effets. Des lectures originales des rythmes, des espaces et des infrastructures de l'énergie sont présentées afin de suivre les transformations à venir et de leur donner de nouvelles significations écologiques. 


\section{Transformations énergétiques sous contrainte écologique forte Alain Nadai \& Grégoire Wallenborn}

L’urgente nécessité de contenir les effets du changement climatique afin de préserver une Terre habitable est aujourd'hui reconnue. Dans son rapport publié en octobre 2018, le GIEC est sorti de sa réserve scientifique pour en appeler à une transformation énergétique (effaçant ainsi la notion un peu trop tranquille de «transition énergétique»), de manière à réduire d'au moins $55 \%$ les émissions de gaz à effet de serre au niveau global d'ici 2030. Ceci suppose un effort plus grand encore dans les pays avancés, des changements profonds dans nos modes de vies ainsi que dans nos manières de penser et de pratiquer notre relation à l'énergie, sa production et ses usages.

\section{Changements de positions}

Au niveau national, des déplacements sont certes en cours dans la fabrique des politiques publiques. En France, en 2012, un débat national sur notre devenir énergétique (DNTE 20122013) a été pour la première fois conduit sur la base des scénarios et visions d'acteurs. Il a constitué un moment d'explicitation politique. En effet, les représentants d'intérêts économiques se sont, pour la première fois explicitement et publiquement, positionnés en défaveur de visions de sobriété énergétique. Ils préferrent une transition énergétique fondée sur l'innovation et l'efficience énergétique, laissant nos visées de croissance et nos modes de consommation quasi inchangés. Une nouvelle génération d'experts est apparue, certes issue en partie des grands corps mais aussi, pour certains, active dans des milieux associatifs et adossant la vision de l'intérêt public à un pluralisme plus ouvert. Ce débat, volontairement peu médiatisé, a de plus constitué un moment décisif de légitimation du scénario Négawatt, jusqu'alors considéré comme marginal et peu sérieux. II s'agit d'un scénario associatif qui émane d'un réseau 


\section{Majeure Transformations énergétiques}

d'ingénieurs et procède d'une vision engagée, opérationnalisée en entrant par la description matérielle du futur. Il contient des options explicites de changement de modes de vie (partage sur le logement ou le transport) faisant valoir une réduction importante de la consommation d'énergie, afin de soumettre les décisions économiques à nos choix de futur. Le DNTE, enfin, a nourri méthodologiquement des processus internationaux tels que la COP21, dans l'idée et la manière de partir des visions d'acteurs - les fameux Intended Nationally Determined Contributions (INDCs) - et en aidant les États à construire ces visions nationales via des projets européens. Plus récemment, c'est le processus d'élaboration de la deuxième Stratégie Nationale Bas Carbone (SNBC2, 2017-2018), qui a été réorienté, dans le sillage du sommet de Paris (2015) et du Plan Climat (2017) de Nicolas Hulot, vers un objectif de neutralité carbone à 2050, induisant des ruptures de méthode symptomatiques de déplacements d'un système techno-politique: nécessité de recalage des grands modèles macro-économiques incapables de simuler cette perspective, interrogation collective à nouveaux frais sur les coûts de décarbonisation de l'économie française. Et aujourd'hui, au moment où nous écrivons, se tient la Convention Citoyenne pour le Climat, dont il sera intéressant de suivre les effets.

\section{Insuffisances, décalages, mouvements}

Si les lignes semblent ainsi bouger au sein de la fabrique des politiques publiques, les déplacements de visions et de méthode ne trouvent pas leur traduction concrète dans les politiques publiques. Les décalages sont mêmes de plus en plus abyssaux entre ce qu'on devrait faire et ce qu'on ne fait pas, le plus dangereux étant, comme le pointe entre autres Greta Thunberg, de faire comme si l'on faisait sans réellement mettre en œuvre les nécessaires changements.

Ces décalages grandissants se lisent notamment dans les formes de revendications, d'actions, de manifestations publiques à la croisée des enjeux climat-énergie. Le contenu et les formes de politisation évoluent fortement à mesure que devient indiscutable et effarante l'impuissance collective à répondre à l'enjeu climatique: extension à la jeunesse (grèves/manifestations des collégiens), désobéissance civile (Extinction Rebellion). Ce constat vaut aussi en partie dans le champ de l'énergie. Par exemple, la ZAD Amassada, démantelée par la police en octobre 2019, porte une opposition à l'éolien industriel en Sud Aveyron qui de par sa visée systémique contraste radicalement d'avec les oppositions riveraines à l'éolien. Si le collectif Amassada reprend des arguments classiques de ces oppositions (bruit, nuisances locales), il est aussi, par bien des aspects (critique du capitalisme, de la finance, de la logique transnationale/globale des infrastructures, des intérêts globaux, de la négation du local, recherche d'une autre forme d'organisation politique...), plus proche de collectifs tels que Tarnac, ou encore la ZAD de Notre Dame des Landes.

En clair, en dépit des déplacements de lignes au cœur de la fabrique des politiques publiques, ou dans les discours, le fait de ne pas avoir fait ce qu'on aurait dû faire appelle aujourd'hui des transformations radicales, ici appelées «transformations énergétiques sous contrainte écologique forte». Au moins deux visions opposées s'affrontent dans ces transformations. D'une part, les États entendent garder la maîtrise de la question éner- 


\section{Multitudes 77}

gétique en étendant les réseaux de distribution de l'énergie - comme en témoigne le projet de la Commission européenne de constituer un grand réseau de transport de l'électricité qui connecterait les éoliennes de la mer du Nord, le photovoltaïque des pays méditerranéens et les stockages hydrauliques des Alpes et de la Norvège. D'autre part, toute une série d'actions locales ont lieu autour de la réappropriation collective des moyens de production d'énergie: réseau des régions $100 \%$ renouvelables en Allemagne, réseau des territoires à énergie positive (CER-Réseau pour la Transition Énergétique) ou mouvement Énergie Partagée en France, renewable energy communities au Royaume Uni, Convention des maires dans l'Union Européenne. Ces initiatives se sont démultipliées au cours des deux dernières décennies, et notamment après l'échec des négociations entre États au sommet de Copenhague (2009), mais elles connaissent depuis quelques années des réorientations et des revers importants avec le passage à des politiques de soutien d'inspiration néolibérale: passage des tarifs d'achat aux appels d'offres, passage de soutien par subvention à des aides remboursables conditionnées à l'élaboration d'un modèle d'affaires... Les analyses récentes attestent de la difficulté de ces réseaux et petites initiatives à se développer dans ces environnements plus concurrentiels. La question se pose donc du devenir de ces modes d'action locale, tout autant que de savoir si ces deux visions sont complémentaires ou incompatibles, notamment au vu des ressources disponibles (minéraux, espace, temps ).

\section{La fin des transitions}

Les «transformations énergétiques sous contrainte écologique forte» désignent dans ce contexte l'idée que la notion opérationnelle de transition énergétique, désignant implicitement le passage d'un état $\mathrm{A}$ (actuel) à un état $\mathrm{B}$ (meilleur), possiblement anticipable (voire linéaire, calculable) dans un monde relativement stable, semble obsolète.

L'idée que nos sociétés modernes sophistiquées pourraient être fortement affectées sous le stress d'un monde à plus de $2^{\circ} \mathrm{C}$ s'est construite depuis quelques années à la croisée de notre incapacité à répondre au changement climatique et de travaux, datant pour certains des années 1980, sur l'effondrement des sociétés (Jared Diamond). Elle est aujourd'hui relayée par divers réseaux militants, au cœur de mouvements politiques, de visions de think tank, et débattue dans divers milieux. L'effondrement, outre des visions de montée extrême des températures et de planète étuve $\left(+7^{\circ} \mathrm{C}\right)$ conduisant à la fin de la plupart des espèces vivantes, signifierait dans des scénarios plus modérés de monde à plus de $2^{\circ} \mathrm{C}$, que les États ne pourraient plus assurer la fourniture stable de services infrastructurels qui se dégraderaient, progressivement ou par rupture, déstructurant nos sociétés et conduisant à des temps de rationnement de fait, de désordres sociaux, d'insécurité... La limite des visions de transitions en environnement stable ne signifie pas pour autant une croyance en l'effondrement. Elle reflète simplement le fait que nous ne pouvons plus rationnellement espérer nous maintenir sous les $2^{\circ} \mathrm{C}$. Nos anticipations étant de plus en plus incertaines, il nous faut organiser une résilience adaptative, c'est-à-dire une capacité à s’adapter qui évolue avec les effets des dégradations. 


\section{Majeure Transformations énergétiques}

La contrainte écologique forte désigne donc, dans cette situation, non seulement l'urgence à réduire nos émissions à court terme mais aussi celle à construire des modes de vie qui nous laissent espérer de pouvoir réduire notre empreinte environnementale pour stabiliser, à moyen terme, un nouveau régime climatique et des espaces de biodiversité riche. Elle implique de trouver les bonnes échelles pour agir et construire des récits à ces fins. Les modifications doivent être profondes et rapides, car le temps d'apprentissage qui nous est offert semble peu compressible. L'urgence climatique ne saurait pour autant justifier la soumission des expériences locales à un impératif technocratique, sous peine de verser dans la barbarie. Isabelle Stengers entrevoyait «la barbarie qui vient » comme le moment où nous serions obligés, faute d'anticipation, d'en passer par des mesures que nous n'aurions jamais acceptées en des temps plus stables. La contrainte écologique forte est aussi ce moment présent, ce seuil avant la barbarie, où le basculement écologique est certes en vue mais où les expériences déjà présentes peuvent encore nourrir des décisions éclairées. Dit autrement, nous ne pouvons plus réfléchir à la qualité des processus de changement sans penser dans le même temps leur possible montée en échelle, mais nous pouvons encore réfléchir aux qualités des montées en échelle que nous connaissons ou entrevoyons.

\section{Qualités, ontologies et sentiers pour une montée en échelle}

La montée en échelle des expériences collectives autour des dispositifs énergétiques pose la question de l'articulation de qualités à une quantité. Par exemple, si l'on prend l'empreinte environnementale comme indicateur quantitatif de l'enjeu énergétique, la question est celle de l'agrégation des multiples ressources engagées dans le développement des nouvelles énergies: énergies fossiles pour construire les dispositifs et infrastructures, ressources minérales (notamment terres rares), espaces, faune, flore, jusqu'aux énergies «sociales», au sens de propension à s'engager dans des processus collectifs qui soient à même de porter ces changements. Il $\mathrm{y}$ a donc une tension entre le besoin de se relier et de se compter (constitution du collectif) et celui de monter en échelle (transformer les espaces-temps), qui renvoie à deux enjeux.

D’une part, comme le souligne Anna Tsing, le capitalisme a besoin de modes de montée en échelle qui s'affranchissent des singularités afin de pouvoir unifier, concentrer et accumuler la richesse (un de ses ressorts) ${ }^{1}$. La scalabilité désigne cette recherche d'une expansion sans obstacles, sans changement de cadre et insensible au caractère indéterminé des rencontres, des matérialités qui sont au cœur des expériences collectives. Cette insensibilité peut être lourde de risques. Au premier rang vient le risque d'une "fossilisation» des nouvelles ressources énergétiques ${ }^{2}$, au sens d'un mode d'exploitation qui reproduise les méthodes, valeurs et savoir faire à la base de l'exploitation des ressources fossiles, si ce sont exclusivement les acteurs et institutions des énergies fossiles qui s'emparent de ces nouvelles

1 Le Champignon de la fin du monde. Sur les possibilités de vie dans les ruines du capitalisme, Les Empêcheurs de penser en rond/ La Découverte, 2015.

2 Raman, Sujatha. «Fossilizing Renewable Energies.», Science as Culture 22, n² (2013), p. 172-180. 


\section{Multitudes 77}

énergies. S'il est une leçon que nous enseignent la sociologie des sciences et techniques (Akrich, Latour) et la philosophie des techniques (Simondon), c'est bien que la technologie, en se développant, engage et recompose le monde autour d'elle. Elle requalifie, alloue des potentialités et des capacités d'action aux multiples ressources qu'elle assemble, sans parler de la manière dont l'espace et le temps n'ont cessé d'être remodelés au fil des innovations technologiques. Les chemins et les destinations peuvent donc être multiples, les potentiels aussi. La qualité peut ici se formuler comme le souci de prendre en compte et de faire compter dans les arènes où se décident les orientations, toutes les entités dont le devenir est engagé par ces processus. Comme le souligne Noortje Marres, la grande difficulté réside dans les jeux de relations qui ne sont jamais clairs, surtout s'il convient de convoquer aussi des non-humains ${ }^{3}$. Le problème ne se résume jamais à mettre des parties bien définies en présence, la définition même de ces dernières, des effets qu'elles subissent ou génèrent, est bien souvent le premier enjeu. Il faut donc se donner avant tout les moyens de s'accorder sur qui nous sommes et ce que seraient nos devenirs.

D'autre part, un second enjeu des transformations énergétiques porte sur les sentiers à emprunter, étant entendu qu'ils sont doublement irréversibles: du fait des infrastructures construites qui verrouillent les devenirs et de la production d'entropie associée à toute activité. On doit donc s'interroger sur ce que pourraient être les sentiers les moins dispendieux. Les énergies dites renouvelables ont un statut très ambigu de ce point de vue. En effet, elles sont supposées reposer sur des énergies de flux (vent, courant, différences thermiques) qui se renouvellent grâce à des cycles naturels (climat, cycle de l'eau), ultimement nourris par l'énergie du soleil. Les énergies sont dites renouvelables tant qu'elles n'épuisent pas les flux sur lesquels elles reposent. Mais cette définition est insatisfaisante car la constitution de ces flux en ressources énergétiques renouvelables convoque bien d'autres ressources. Nous n'avons que des connaissances très partielles de ce qu'est le bilan entropique matièreénergie d'une éolienne ou d'un panneau solaire, par exemple. Le mouvement «low tech» tente de répondre à ces questions en proposant des dispositifs sans doute moins « efficients » mais plus robustes et réparables ${ }^{4}$. Toutefois la distinction entre «high tech» et «low tech» ne tient que comme tension à soumettre au collectif qui se constitue. En effet, toute low tech repose in fine sur un réseau de technologies qui brouille cette distinction.

Nous pensons que la montée en échelle des expérimentations collectives est à la fois urgente et pleine de dangers. Le passage d'une échelle à l'autre est en soi une expérimentation qui appelle la constitution d'un collectif approprié - celui-ci ne peut résulter de l'injonction de l'État ou de la prescription du marché. Ce collectif est seul à même de faire valoir les multiples qualités des dispositifs locaux et de négocier ce qui est scalable ou ne l'est pas. Quels sont les temps de ces processus? Quelles lectures devons-nous en opérer pour en lire les qualités, en comprendre les temporalités, et en orienter les finalités? Quelles leçons pouvons-nous tirer des multiples expériences déjà engagées à divers niveaux et dans diverses aires culturelles? 


\section{Majeure Transformations énergétiques}

\section{Quelques contributions}

Les contributions à cette Majeure proposent des points de vue variés qui permettent de mieux cerner les enjeux théoriques et pratiques des nécessaires transformations énergétiques. Les plus théoriques introduisent des concepts que nous pouvons voir à l'œuvre dans les autres textes.

Gordon Walker propose une analyse rythmique afin de saisir la production et la reproduction des pratiques énergétiques dans leurs articulations temporelles, spatiales, sociales et technologiques. Partant de l'idée que toute entité possède un rythme qui est lié aux rythmes d'autres entités, il considère que le rythme est ce qui permet de penser l'interconnexion entre énergie, société et climat, qui se fait en un lieu donné avec un certain taux de dépense énergétique. Selon cette perspective, les infrastructures énergétiques organisent le temps et l'espace des rythmes-collectifs mais sont elles-mêmes reproduites par une série de rythmes (d'extraction des énergies fossiles, de leurs distributions, de leurs consommations et de leurs émissions). Walker note que tous ces rythmes sont fortement coordonnés dans les sociétés modernes, ce qui explique les fortes résistances au changement qui sont de fait des déstructurations des rythmes actuels dans un premier temps. En contrepoint, il indique que les personnes qui vivent «horsréseau» le font selon des rythmes plus lents, plus aléatoires puisque ces rythmes dépendent de l'énergie disponible via des sources variables (solaire ou vent). Dans la mesure où les rythmes du climat se modifient, les nouveaux rythmes branchés sur des sources plus variables seront plus lents, plus erratiques, mais ils laisseront plus de place aux corps, à la diversité des vies humaines et aux potentiels singuliers logés dans les agencements locaux.

Olivier Labussière nous invite à descendre l'échelle spatiale, à sortir d'un abord de l'espace comme simple réceptacle des énergies éoliennes et solaires, pour s'intéresser aux mailles où s'agencent les dispositifs de capture d'énergie avec des usages, toujours pris dans des configurations socio-historiques particulières. Il avance que la minimisation de l'entropie (ce qui inclut la dispersion des ressources minérales) ne peut être optimisée qu'en analysant finement les relations entre énergie, matière et espace. Ainsi la récupération du flux solaire notamment ne doit pas se penser uniquement en superficies mais en termes de «milieu associé», concept qu'il reprend à Simondon et qu'il étend au collectif des humains et non humains qui rend concret le dispositif technique. La question de la "part récupérable» des flux d'énergie doit être articulée aux manières dont le dispositif crée son tissu de relations aux entités environnantes, d'où pourra éventuellement émerger son fonctionnement concret. Une capture sociotechnique réussie témoigne de la création d'une maille viable c'est-à-dire d'un collectif qui a pu intégrer le dispositif à son ensemble de relations et de pratiques.

Fanny Lopez constate que les infrastructures caractérisées par une organisation centralisée (réseau routier, réseau électrique ) sont en piteux état, fruit d'un désinvestissement sous les politiques néolibérales. Elle remarque également que les infrastructures façonnent le mode de production et de consommation, et qu'il s'agit donc d'en changer si l'on prend au sérieux l'impératif écologique. Les infrastructures jouent un rôle important dans la constitution des liens entre les rythmes et les mailles au niveau local. La tentation est donc grande de construire des communautés basées sur des énergies renouvelables locales et qui soient les plus autonomes possible des grands réseaux. Un nouveau paysage se dessine alors dans lequel les régimes socio- 


\section{Multitudes 77}

techniques seraient plus éclatés, plus éparpillés et plus résilients localement. Néanmoins, la question se pose de la solidarité entre les territoires bien pourvus en ressources renouvelables et ceux qui le sont moins (notamment les villes). Mais, en tout état de cause, on ne fera pas l'économie d'une discussion collective sur les infrastructures nécessaires au maintien des activités considérées comme vitales et sur le choix d'en ranger certaines au patrimoine historique de l'anthropocène.

Edith Chezel nous emmène en Frise du nord, à la frontière entre l'Allemagne et le Danemark, là où se sont constitués des champs d'éoliennes gérés en coopérative depuis les années 1990. Ces éoliennes transforment non seulement le paysage mais aussi le régime de sentir, penser et agir au travers des turbulences rythmiques que traverse le collectif cherchant à s'approprier le vent et les nouveaux flux financiers. L'article insiste sur l'épaisseur des expériences sensibles, pratiques et politiques du collectif à la fois comme mémoire, constitution de ses valeurs, et comme surplus des expériences, potentiel pour reconfigurer le collectif au fur et à mesure qu'il s'étend. Chezel conclut que l'apprentissage et la reconfiguration du collectif autour des nouvelles sources d'énergie prennent un temps, qui semble peu compressible - alors que nous avons de fait peu de temps pour généraliser ces expériences.

Antoine Fontaine montre de son côté les difficultés que les projets collectifs autour de l'énergie photovoltaïque ont rencontrées en France. Les politiques publiques françaises ont en effet d'abord octroyé les subsides aux propriétaires qui installent des panneaux photovoltaïques sur leur toiture, ce qui rend par comparaison plus onéreux les projets collectifs. Fontaine s'intéresse alors à des collectifs qui ont pu contourner les obstacles de ces politiques individualisantes: les centrales villageoises, nées dans la région Rhône-Alpes dans les années 2010. Il montre l'importance de la mise à disposition de cadres juridiques alternatifs, qui représente une forme de montée en échelle par essaimage et partage de ressources. Outre les questions d'organisation inhérentes à tout collectif, l'article montre tout l'intérêt d'assembler des citoyens autour de sources d'énergie locales, notamment en termes de justice sociale et de prise de conscience de leurs usages, de telle sorte que la ressource solaire soit construite comme commun. Les communautés d'énergie apparaissent comme une voie d'apprentissage pour modifier les rythmes de consommation et les rendre plus adéquats à la production.

Jim Segers nous présente la création d'un dispositif d'intervention en milieu urbain afin de constituer une communauté d'énergie. Fort de son expérience dans d'autres projets qui coalisent des acteurs hétérogènes autour d'enjeux urbains communs, il présente une méthodologie pour affronter les «questions délicates» qui allient construction d'un prototype matériel et construction du collectif autour de ce prototype. Selon lui, les problèmes intriqués (wicked problems), tels que ceux face auxquels nous met le changement d'énergie, demandent une action pratique et située pour trouver leur issue; un argument qui plaide en faveur de processus fortement territorialisés.

Elvan Arik, Sylvy Jaglin et Éric Verdeil soulignent l'influence des configurations sociales et politiques pour les transformations énergétiques urbaines. Les villes se sont progressivement constituées autour des infrastructures énergétiques, tout en modifiant les normes de confort et en accélérant les rythmes sociaux. Les deux cas présentés (réseau de distribution du gaz à Istanbul et développement du photovoltaïque au Cap) montrent que 


\section{Majeure Transformations énergétiques}

l'accès aux infrastructures est très inégal dans un régime (néolibéral) où le prix de l'énergie est le paramètre dominant. Les plus démunis recourent à des solutions hybrides qui font appel à diverses sources d'énergie sans toujours garantir l'accès suffisant aux services énergétiques. Si le réseau a été conçu comme instrument de solidarité entre les ménages et entre les territoires, les dispositifs économiques des politiques publiques modulent de fait cette solidarité au profit de ceux qui peuvent s'offrir les nouvelles technologies. L'article souligne l'importance des récits locaux pour la reconfiguration des systèmes énergétiques. Les cas étudiés s'inscrivent dans des récits sociopolitiques en fonction des situations locales. Dès lors qu'un déploiement à large échelle est nécessaire, le politique doit donner du sens à la reconfiguration proposée, les gouvernements cherchant alors diverses justifications aux investissements consentis, en les raccrochant évidemment à leur idéologie (souvent sous les auspices de la modernisation). Quels nouveaux récits politiques peuvent assurer les nouvelles formes de solidarités qui devront accompagner le déploiement de sources locales d'énergie? Les auteurs invitent aussi à relativiser ("provincialiser») les récits et impératifs de transition énergétique émanant des pays occidentaux développés, et répondant à leurs configurations d'intérêts. L'enjeu de développement est bien souvent premier dans les configurations des Suds.

Enfin Nathalie Ortar décrit, au sein d'un projet européen de recherche, les tentatives de créations de coalitions d'acteurs locaux travaillant dans des institutions hétérogènes et qui n'ont pas l'habitude de se parler. Le point commun de départ des discussions était d'intégrer les sciences sociales afin de s'affranchir d'une vision purement technologique des potentiels de transformation. Se plaçant ainsi avant la constitution de «mailles» et la mise en question des rythmes énergétiques, Ortar souligne l'importance d'établir une vision et un langage communs. Elle aussi insiste sur les aspects locaux (à l'échelle d'une ville) irréductibles à toute visée universalisante des transformations énergétiques indispensables.

\section{Modes de vie, ici et ailleurs}

Notre appel à textes n'a pas donné lieu à des propositions portant sur la transformation des modes de vie, pourtant essentielle pour espérer une Terre suffisamment habitable. Les transformations énergétiques ne sont pas seulement affaire de déploiement de sources renouvelables et de moyens de stockage, même articulés à des collectifs volontaristes. Les scénarios qui envisagent un déploiement de $100 \%$ d'énergie renouvelables à l'horizon 2050 et qui tiennent compte des ressources limitées (minerais, espace), prévoient que la consommation d'énergie doit être divisée au moins par deux d'ici là. C'est évidemment une mise en question radicale de l'organisation des rythmes dont il s'agit-Il nous manque des récits pour rendre désirable cette nouvelle société, plus lente, plus en phase avec ce que peuvent soutenir les écosystèmes à un moment donné. Bien sûr, les collectifs se multiplient qui optent pour des modes de vie décroissants, qui contestent la notion de confort, mais leurs articulations à d'autres échelles spatio-temporelles sont problématiques. Quelles sont les activités et les infrastructures à éliminer, celles qui sont nuisibles et nocives? Que devient la polyrythmie socio-écologique lorsqu’on réduit le nombre des activités? Quelles transformations peuvent se réaliser à l'échelle globale en s'appuyant sur les expériences décrites ici? 